\title{
Bases para el establecimiento de las Ciencias Aeronáuticas en la Facultad de Ciencias Espaciales de la Universidad Nacional Autónoma de Honduras
}

Omri Amaya

\section{Resumen}

Esta publicación refleja lo esencial del trabajo de investigación desarrollado por un Equipo Interinstitucional integrado por representantes de la Dirección de Aeronáutica Civil (DGAC) y la Asociación para el Desarrollo Aeronáutico y Educativo de Honduras (ADAEH) junto con profesionales de la Universidad Nacional Autónoma de Honduras (UNAH).

El panorama general de la investigación sitúa el desarrollo de la aeronáutica civil en Honduras en el contexto del desarrollo internacional del rubro, enfatizando en su estatus científico, los contenidos con los cuales se forma a los profesionales del campo, su evolución histórica, las características propias de la aeronáutica en Honduras y, la oferta y la demanda existente de profesionales aeronáuticos a nivel mundial.

Los resultados de esta investigación han orientado el posterior desarrollo del Departamento de Ciencias Aeronáuticas en la Facultad de Ciencias Espaciales, influyendo sobre la definición de los recursos necesarios para el funcionamiento del Departamento, la elaboración del diagnóstico para la Carrera de una Licenciatura en Ciencias Aeronáuticas y la composición del su Plan de Estudios y Plan de Sostenibilidad; así como en el montaje del Primer Diplomado en Gestión de Sistemas Aeroportuarios.

Palabras clave: Bases, Ciencias Aeronáuticas, Aeronáutica Civil, perfil del profesional aeronáutico, oferta académica.

\section{Abstract}

This publication reflects the essence of the research developed by an interagency team composed of representatives of the Direction of Civil Aviation (DGCA) and the 
Association for the Aeronautical Development and Education of Honduras (ADAEH) together with professionals from the National Autonomous University of Honduras (UNAH).

The overview of research puts the development of civil aviation in Honduras in the context of international development for the category, emphasizing its scientific status, the contents of which is formed by professionals of the field, its historical evolution, the characteristics of aeronautics in Honduras and offer and the demand for aviation professionals worldwide.

The results of this research have guided the subsequent development of the Department of Aeronautical Sciences at the Faculty of Spatial Sciences, influencing the definition of resources necessary for the functioning of the Department, the development of diagnostics for the Career of a Bachelor of Aeronautical Science and the composition of the Curriculum and Sustainability Plan; as well as the construction of the First Diploma in Management Airport Systems.

Keywords: Bases, Aeronautical Science, Civil Aviation, aeronautical profile professional, academic offerings.

Omri Amaya, (Omri.amaya@unah.edu.hn) Departamento de Ciencias Aeronáuticas,Facultad de Ciencias Espaciales,Universidad Nacional Autónoma de Honduras 


\section{Introducción}

El documento "Bases para el establecimiento de las Ciencias Aeronáuticas en la Facultad de Ciencias Espaciales de la Universidad Nacional Autónoma de Honduras" establece una perspectiva amplia de la aeronáutica civil en el contexto nacional e internacional abordando puntos focales del campo, entre los que se pueden mencionar: el concepto de Aeronáutica, su definición, Tendencias y Perfil Profesional de quienes la ostentan como carrera; un análisis situacional de la problemática en el campo aeronáutico de Honduras, en la que se visualizó un espacio con gran potencial para la Universidad; las políticas aeronáuticas de Estado cuyo germen se encuentra la Ley de Aeronáutica Civil vigente; las orientaciones y limitaciones que ofrece el Plan de Nación para el desarrollo del rubro; algunos elementos de la evolución de la aeronáutica y el lugar que ocupa en este campo la región; las estadísticas disponibles en la DGAC de profesionales comparada con la infraestructura y las aeronaves disponibles, entre otros.

Dada la amplitud de temas abordados en el citado documento Bases y la limitación de espacio, en este artículo nos limitaremos a mencionar los elementos más importantes del mencionado documento. El artículo inicia presentando los objetivos que se propone; seguidamente, se resume la metodología aplicada la cual utilizó fuentes de información primarias y secundarias; luego se resaltan tres de los resultados presentados en el documento Bases: un perfil del profesional y los técnicos aeronáuticos partiendo de lo establecido por la $\mathrm{OACl}$ y por la literatura consultada; un ejercicio de bench marking llevado a cabo con cuatro universidades del continente americano definiendo el conjunto de asignaturas propuestas en cada caso para formar técnicos y profesionales aeronáuticos con el grado universitario; un resumen de las preferencias y opiniones expresadas por una población integrada por profesionales aeronáuticos, estudiantes de escuelas de vuelo certificadas, profesionales Universitarios y estudiantes de último año de colegios de educación secundaria, públicos y privados y, empresas aéreas, esta población fue consultada mediante una entrevista estructurada.

En la última parte del artículo se exponen, de forma concisa y ordenada las conclusiones del trabajo realizado y las recomendaciones analizadas, discutidas y consensuadas por el Equipo interinstitucional responsable último de la elaboración del documento Bases.

El Documento Bases es un primer paso en firme para el desarrollo del campo de la aeronáutica civil en Honduras desde una perspectiva civil. Su intención última es el establecimiento de una plataforma de diálogos y consensos para 
construir sobre la base de los aportes científicos de la Academia, el campo de la aeronáutica civil de Honduras con identidad propia.

\section{Objetivos}

\section{Objetivo general}

Establecer las bases para que el campo de las Ciencias Aeronáuticas, particularmente la Aeronáutica Civil, se organice y establezca en la Facultad de Ciencias Espaciales (FACES), justificando de esta manera la incursión de la UNAH como apoyo directo al desarrollo de un campo considerado vital para el Desarrollo social y económico de Honduras.

\section{Objetivos específicos}

- Definir el campo Aeronáutico, sus tendencias pasadas y actuales, analizando los perfiles profesionales de planes de estudio y de egresado de varias universidades la mayor parte de ellas estadounidenses y de renombre en la aviación continental.

- Analizar la problemática de la Aeronáutica Civil en Honduras, partiendo del contexto regional socioeconómico, político y educativo hasta llegar al propio campo de estudio, incluyendo como elementos relevantes de análisis la educación aeronáutica militar y las oportunidades y limitaciones que tienen la actual Ley de Aeronáutica Civil y el Plan de Nación.

- Presentar elementos de la Oferta y Demanda para el establecimiento del campo de la Aeronáutica Civil en la UNAH, tomando en consideración diferentes sectores de la población hondureña, incluyendo personas sin conocimiento del campo y empresas y profesionales activos en el rubro.

\section{Método}

La investigación ofrece un estudio exploratorio cualitativo que fundamenta sus resultados en datos cuantitativos extraídos de la literatura y sitios web investigados. 
El proceso de elaboración del documento Bases del cual este artículo expone los principales aportes, se puede dividir en las siguientes fases:

- Consulta bibliográfica que incluyó títulos sobre historia de la aviación, la clasificación de la Aeronáutica como ciencia, el marco jurídico y reglamentario nacional e internacional, Ley de Plan de Nación y Visión de País y distintos documentos oficiales de análisis de la coyuntura aeronáutica mundial;

- Repaso de contenidos para la formación en ciencias aeronáuticas que incluyó la revisión de la oferta formativa en materia aeronáutica de un grupo de universidades del continente americano utilizando la técnica de Bench Marketing;

- Aplicación de una encuesta a profesionales y no profesionales interesados reales o potenciales en campo aeronáutico y a empresas aeronáuticas, se entrevistó a cinco grupos como lo muestra la tabla 1; 4. Presentaciones periódicas al equipo interinstitucional de los resultados obtenidos en el proceso de investigación.

\begin{tabular}{|c|c|c|c|}
\hline No. & Sector de la Población & $\begin{array}{l}\text { Personas } \\
\text { Encuestadas }\end{array}$ & Cantidad \\
\hline 1 & Profesionales aeronáuticos & $\begin{array}{l}\text { Personal Técnico } \\
\text { Aeronáutico }\end{array}$ & 92 \\
\hline 2 & $\begin{array}{l}\text { Estudiantes de las Escuelas de } \\
\text { Vuelo Certificadas }\end{array}$ & $\begin{array}{l}\text { Estudiante Escuela } \\
\text { de Aviación }\end{array}$ & 13 \\
\hline 3 & Profesionales Universitarios & $\begin{array}{l}\text { Profesional } \\
\text { Universitario }\end{array}$ & 81 \\
\hline 4 & $\begin{array}{l}\text { Estudiantes de último año de co- } \\
\text { legios de Educación Secundaria, } \\
\text { públicos y } \\
\text { privados }\end{array}$ & $\begin{array}{l}\text { Estudiantes de } \\
\text { Educación Media }\end{array}$ & 40 \\
\hline 5 & Empresas aéreas & Funcionarios & 17 \\
\hline$\frac{\text { Total Personas }}{\text { Entrevistadas }}$ & & 243 & \\
\hline
\end{tabular}

Tabla 1. Personas encuestadas

Fuente: Datos recolectados a través de los instrumentos de investigación

Utilizados durante el estudio para el documento Bases. 


\section{Resultados}

\section{- Perfil profesional aeronáutico}

Según la OACI las especialidades de personal con mayor demanda en la actualidad son los pilotos, el personal de mantenimiento aeronáutico y los controladores de tránsito aéreo. Sin embargo, existe un abanico variado de profesiones dentro del ámbito aeronáutico, el documento Bases da cuenta de las siguientes: 1) Piloto que es el designado por el explotador o por el propietario de una aeronave en el caso de la aviación general, para estar al mando y asumir la responsabilidad de la operación segura de un vuelo; 2) Personal de mantenimiento, como encargados de vigilar la aeronavegabilidad de la aeronave; 3 ) despachador de vuelo quien supervisa todo lo necesario para que un vuelo pueda salir incluyendo mapas meteorológicos, rutas de vuelo, documentación, zonas de aproximación, entre otros; 4) tripulantes de cabina, cuya función es garantizar que se cumplan los procedimientos de seguridad en el avión, tanto en procedimiento normal, como en caso de emergencia. Además, el tripulante de cabina es el encargado de la atención al público en una situación crítica; 5) Personal de rampa, encargado de brindar sus servicios en un área definida para el acomodamiento de las aeronaves, con el propósito de embarque y desembarque de pasajeros y/o carga, reabastecimiento de combustible, limpieza y estacionamiento; 6) Meteorólogos, encargados de comprender, explicar, observar o predecir los fenómenos de la atmósfera terrestre y el modo en que éstos afectan a la vida sobre el planeta; 7); Personal gubernamental, quienes son la autoridad del estado dentro del Aeropuerto con funciones de migración, sanidad y pago de impuestos; 8) Bomberos aeroportuarios. 9) Controlador de Tránsito Aéreo, que atienden la función de controlar el del tránsito aéreo a los diferentes niveles de acuerdo al espacio aéreo designado por la autoridad aeronáutica; 10) Personal de Seguridad Aeroportuaria, quienes en los aeródromos establecen las medidas y procedimientos a ser aplicados tanto en tierra como en vuelo para resguardar y proteger aeronaves, pasajeros, carga, correo y suministros; 11) Personal de mantenimiento de instalaciones aeroportuarias encargado de la infraestructura aeroportuaria en funciones de mantenimiento tanto en carpintería, telefónica, albañilería, electricidad y de seguridad industrial.

\section{- Oferta formativa en Universidades del Continente}

Se toman en consideración es este acápite solamente aquellos centros de formación que cuentan con carreras en materia de ciencias aeronáuticas en el Grado de Licenciatura u otros grados similares. Se constata que las orientaciones de formación son Piloto Aviador, Mecánico de Mantenimiento de Aviones y Contro- 
lador de Tránsito Aéreo, estos tres núcleos de formación se ven complementados con una parte de gerencia de aeropuertos y otro bloque dedicado al diseño y fabricación de aeronaves y componentes. La información disponible se distribuyó entre universidades civiles y militares con una alta prevalencia de las primeras sobre las segundas.

\begin{tabular}{|c|c|c|c|}
\hline Países & $\begin{array}{l}\text { No. Universida- } \\
\text { des }\end{array}$ & Militar & Civil \\
\hline 1. EEUU & 68 & 2 & 66 \\
\hline 2. Brasil & 11 & 1 & 10 \\
\hline 3. México & 9 & 1 & 8 \\
\hline 4. Canadá & 5 & 0 & 5 \\
\hline 5. Argentina & 4 & 1 & 3 \\
\hline 6. Perú & 4 & 0 & 4 \\
\hline 7. Chile & 3 & 1 & 2 \\
\hline 8. Colombia & 3 & 0 & 3 \\
\hline 9. Venezuela & 2 & 1 & 1 \\
\hline 10. República Dominicana & 2 & 1 & 1 \\
\hline 11. Bolivia & 1 & 0 & 1 \\
\hline 12. Ecuador & 1 & 1 & 0 \\
\hline 13. El Salvador & 1 & 0 & 1 \\
\hline 14. Guatemala & 1 & 0 & 1 \\
\hline 15. Honduras & 1 & 1 & 0 \\
\hline 16. Panamá & 1 & 0 & 1 \\
\hline
\end{tabular}

Tabla 2. Número de universidades por países del continente americano que ofrecen el campo aeronáutico.

Fuente: Elaboración propia en base a distintas fuentes.

La gran mayoría de centros de formación, 107 sobre un total de 117, es decir un $91 \%$ de los centros son civiles. Con este último dato vemos que Honduras, al contarse con un solo centro de formación en el ámbito militar, no se está en la tendencia continental. Salta a la vista que Estados Unidos es el país que más domina el campo con 68 centros de investigación y universidades orientadas en el campo, seguida de lejos por Brasil que cuenta con 12 centros; esto convierte al 
país norteamericano en un referente importante para la creación de una carrera en Ciencia Aeronáutica Civil.

Tabla 3. Carreras de Aeronáutica en Centroamérica y el Caribe.

\begin{tabular}{|c|c|c|c|}
\hline País & $\begin{array}{l}\text { Universi- } \\
\text { dad }\end{array}$ & Titulo(s) Obtenido(s) & Especialidad (s) \\
\hline $\begin{array}{l}\text { El Salva- } \\
\text { dor }\end{array}$ & $\begin{array}{l}\text { Universi- } \\
\text { dad Don } \\
\text { Bosco }\end{array}$ & $\begin{array}{l}\text { Técnico en Manteni- } \\
\text { miento Aeronáutico }\end{array}$ & $\begin{array}{c}\text { - Mecánico de } \\
\text { Mantenimiento } \\
\text { de Aviones }\end{array}$ \\
\hline Honduras & $\begin{array}{c}\text { Univer- } \\
\text { sidad de } \\
\text { Defensa } \\
\text { de Hondu- } \\
\text { ras }\end{array}$ & $\begin{array}{l}\text {-Licenciado en Cien- } \\
\text { cias Aeronáuticas } \\
\text { Militares, } \\
\text {-Técnico Universitario } \\
\text { Militar en Manteni- } \\
\text { miento Aeronáutico, } \\
\text {-Técnico Universitario } \\
\text { Militar en Aviónica }\end{array}$ & $\begin{array}{c}\text {-Piloto Aviador } \\
\text { Militar } \\
\text {-Mecánico de } \\
\text { Mantenimiento } \\
\text { de Aviones } \\
\text {-Controlador de } \\
\text { Tránsito Aéreo } \\
\text { Militar }\end{array}$ \\
\hline Panamá & $\begin{array}{l}\text { Universi- } \\
\text { dad Tecno- } \\
\text { lógica de } \\
\text { Panamá }\end{array}$ & $\begin{array}{l}\text {-Licenciatura en Inge- } \\
\text { niería Aeronáutica } \\
\text {-Técnico en Ingeniería } \\
\text { de Mantenimiento de } \\
\text { Aeronaves con Espe- } \\
\text { cialización en Motores } \\
\text { y Fuselajes } \\
\text {-Licenciatura en Admi- } \\
\text { nistración de Aviación } \\
\text { con Opción a Vuelo } \\
\text { (Piloto) } \\
\text {-Licenciatura en Admi- } \\
\text { nistración de Aviación } \\
\text {-Técnico en Despacho } \\
\text { de Vuelo }\end{array}$ & $\begin{array}{c}\text {-Piloto Aviador } \\
\text { - Administración } \\
\text {-Despachador } \\
\text { de Vuelo }\end{array}$ \\
\hline
\end{tabular}




\begin{tabular}{|c|c|c|c|}
\hline $\begin{array}{l}\text { República } \\
\text { Dominica- } \\
\text { na }\end{array}$ & $\begin{array}{c}\text { Academia } \\
\text { Aérea } \\
\text { Gral. Piloto } \\
\text { Frank Feliz } \\
\text { Miranda }\end{array}$ & $\begin{array}{c}\text {-Licenciatura en Cien- } \\
\text { cias Militares y Aero- } \\
\text { náutica }\end{array}$ & $\begin{array}{l}\text {-Piloto Aviador } \\
\text { Militar } \\
\text {-Mecánico de } \\
\text { Mantenimiento } \\
\text { de Aviones } \\
\text {-Controlador de } \\
\text { Tránsito Aéreo } \\
\text { Militar }\end{array}$ \\
\hline $\begin{array}{l}\text { República } \\
\text { Dominica- } \\
\text { na }\end{array}$ & $\begin{array}{l}\text { Academia } \\
\text { Superior } \\
\text { de Cien- } \\
\text { cias Aero- } \\
\text { náuticas }\end{array}$ & $\begin{array}{c}\text {-Técnico Superior } \\
\text { Controlador de Tránsi- } \\
\text { to Aéreo }\end{array}$ & $\begin{array}{l}\text {-Controlador de } \\
\text { Tránsito Aéreo }\end{array}$ \\
\hline Nicaragua & No Existe & - & - \\
\hline $\begin{array}{c}\text { Costa } \\
\text { Rica }\end{array}$ & No Existe & - & - \\
\hline $\begin{array}{l}\text { Guate- } \\
\text { mala }\end{array}$ & $\begin{array}{l}\text { Universi- } \\
\text { dad Gali- } \\
\text { leo }\end{array}$ & $\begin{array}{l}\text {-Técnico Universitario } \\
\text { en Administración de } \\
\text { Empresas de Aviación }\end{array}$ & -Administración \\
\hline
\end{tabular}

Fuente: Elaboración propia, en base a distintas fuentes.

De los 6 técnicos ofrecidos en la región 2 son impartidos en Panamá y 2 en Honduras, en cambio de las 6 Licenciaturas 3 son ofrecidas en Panamá, el único País de la región que cuenta con más de una Carrera en el Grado de Licenciatura. Resalta el hecho de no contar en toda la zona con una maestría, lo cual refleja en cierta medida una laguna en la oferta académica del campo.

El estudio toma como referencia 4 universidades una de las cuales es de Norte América, 2 son de Suramérica y una de Centroamérica. A continuación se presenta de forma resumida la composición de materias para cada una de las carreras ofrecidas 
Gráfico 1: Universidad Embry Riddle EEUU, Bachelor of Science Degree in Aeronautical Science

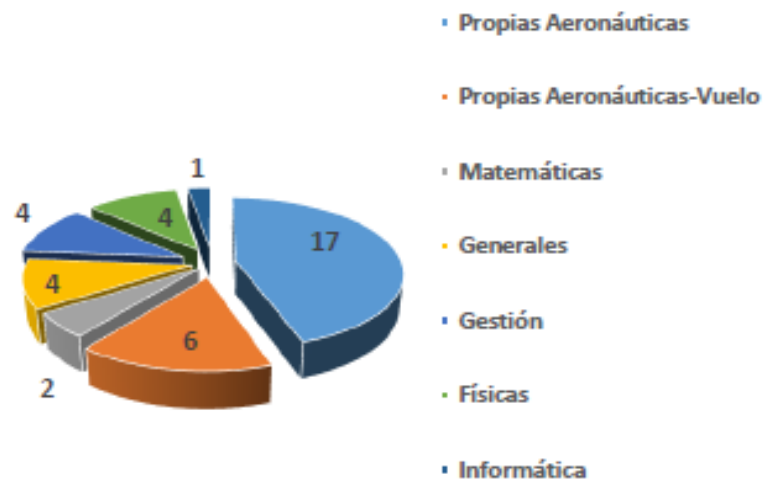

Gráfico 2: Univ. "Fundacão Mineira de Educacãa e Cultura (FUMEC), Brasil. Lic. en Ciencias Aeronáuticas

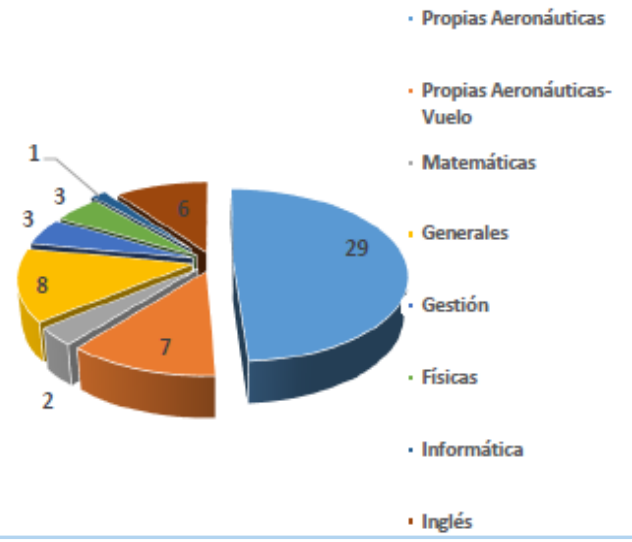

Gráfico 3: Universidad Técnica Federico Santa María, Chile. Ingeniería en Aviación Comercial

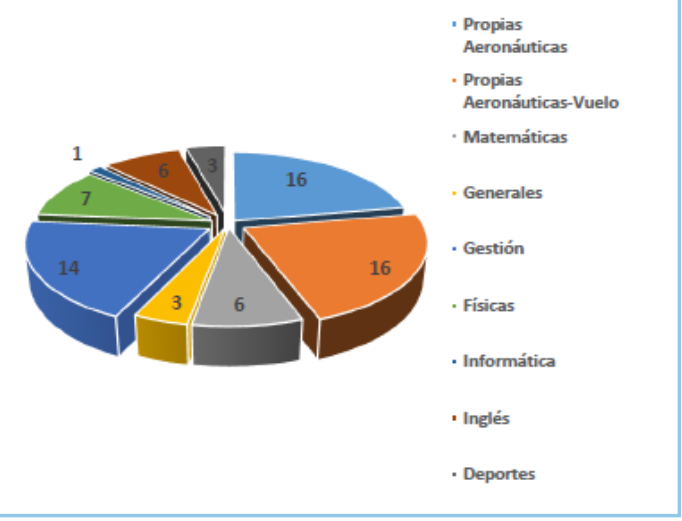


Gráfico 4: Universidad Privada San Martín de Porres, Perú. Licenciatura en Ciencias Aeronáuticas

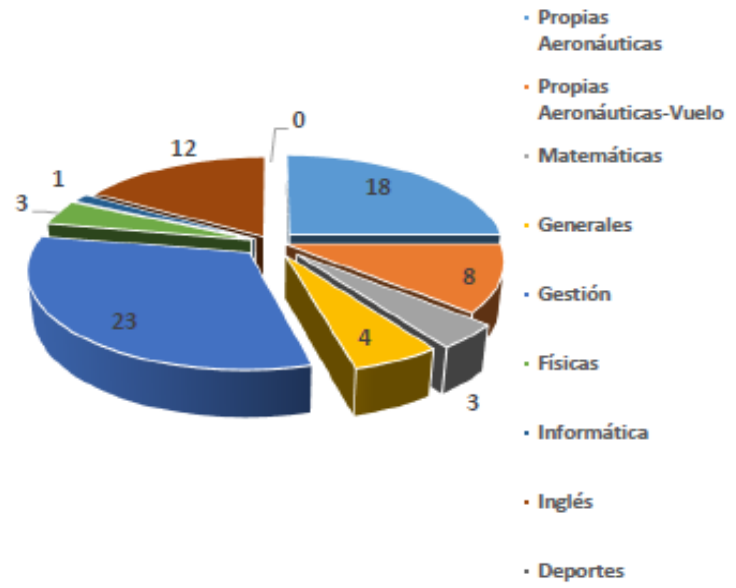

En base a las tendencias de las Carreras Aeronáuticas a Nivel Superior que fueron analizadas, se puede concluir que el perfil de egresado de una carrera aeronáutica responde a las siguientes características:

- Las universidades se proponen formar un profesional con capacidad de diseñar, adaptar, transformar, gerenciar, administrar y regular todo tipo de recursos afines al campo de la aviación sea civil o militar para contribuir al desarrollo económico y social de su país.

- Los egresados de estos programas tienen una sólida formación científica, tecnológica, administrativa y humana, teniendo la capacidad de desempeñarse en campos técnicos, administrativos y gerenciales que requieran liderazgo, alta responsabilidad, máxima idoneidad y confiabilidad profesional logrando en los casos propios la certificación que la entidad regulatoria de aeronáutica nacional aplique según sea el caso.

- Estas carreras dan una sólida formación en ciencias exactas, naturales y básicas que el nivel superior de la educación universitaria amerita.

- Lo anterior se complementa con la formación humana y profesional de los programas que permite mediante la creatividad, liderazgo, iniciativa e innovación, afrontar los problemas técnicos, económicos, sociales y logísticos propios del sector aeronáutico. 
- El currículo de estos planes de estudios brindan a su vez una formación cultural apropiada para el desempeño de una profesión con fuertes nexos en el fenómeno de la globalización, lo que implica el dominio de lenguas extranjeras así como del negocio aeronáutico, claramente internacionalizado hoy en día.

- Preferencias de los entrevistados propios del campo y de fuera del campo aeronáutico

Para la obtención de información sobre la percepción de la demanda de distintos destinatarios potenciales de formación universitaria en el campo aeronáutico se aplicó un cuestionario de seis preguntas a cinco poblaciones distintas: 1) Profesionales Técnicos Aeronáuticos; 2)Estudiantes de Escuelas de Vuelo Certificadas; 3) Profesionales Universitarios; 4) Estudiantes de último año de colegios de Educación Secundaria; 5) Empresas aéreas. Mientras, en el Documento Bases se hace una exposición amplia sobre la metodología aplicada y sobre cada una de las poblaciones entrevistadas, para este artículo se presenta un resumen de las respuestas ofrecidas por todas las poblaciones entrevistadas.

Gráfico 5: ¿Considera Usted importante de la Creación de un Departamento de Ciencias Aeronáuticas?

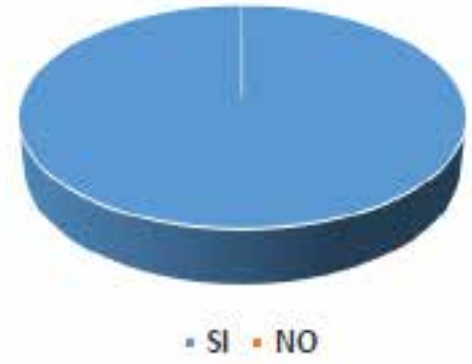

Fuente: Elaboración propia en base al Documento Bases.

Junto con la respuesta a la última pregunta sobre el tema de contratación de egresados de la UNAH, esta respuesta fue de las más contundentes, toda la población considera importante y oportuna la creación de un Departamento de Ciencias Aeronáuticas en la UNAH, esto se corresponde con las lagunas de formación percibidas en lo relacionado con la formación para desempeñarse en Honduras, en 
los rubros de la aeronáutica civil. La respuesta también se hace eco de la tendencia continental a privilegiar la formación civil sobre la militar para el campo aeronáutico.

Gráfico 6: ¿Qué tipo de Educación a Nivel Superior en el Campo de Ciencias Aeronáuticas le gustaría recibir?

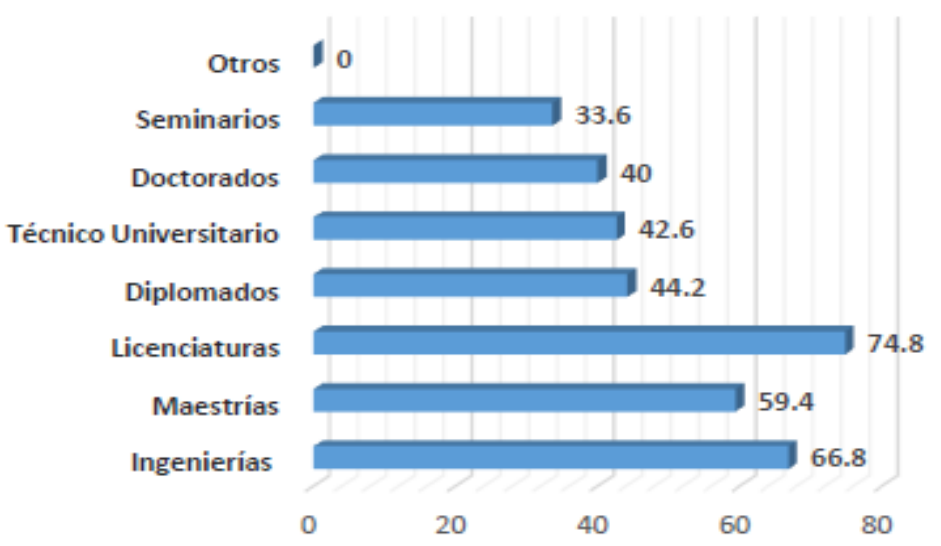

Fuente: Elaboración propia en base al Documento Bases.

Como muestra el gráfico los entrevistados en su conjunto prefieren una Licenciatura como carrera universitaria por encima del resto de propuestas que se pueden hacer desde la Educación Superior. De todas formas al hacer una revisión de los resultados específicos de la consulta se podría ver que de los entrevistados sólo los profesionales universitarios dieron un mayor porcentaje a una ingeniería $(59 \%)$ que a una licenciatura (46). 
Gráfico 7: ¿Con qué orientación aeronáutica le interesaría estudiar?

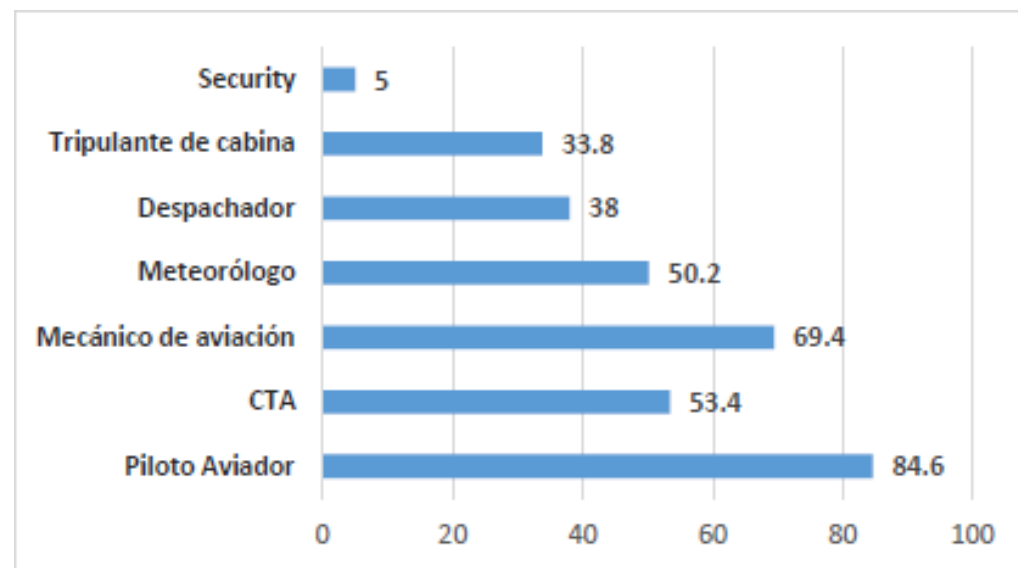

Fuente: Elaboración propia en base al Documento Bases.

En la respuesta a esta pregunta los encuestados se orientaron hacia aquello que resulta más visible en el campo, el piloto aviador (84.6\%) y mecánico de aviación (69.4); el dato se alinea con una tendencia mundial si es leído a la luz de la pregunta 2 que da la mayor importancia al grado universitario en el nivel de Licenciatura, recuérdese que entre las opciones de la segunda pregunta también se encontraban opciones universitaria que no conducen a grado y que, en algunos casos en las universidades se abren para profesionales no egresados de la universidad o para técnicos de campos especializados, pero que sólo cuentan con la formación secundaria. 
Gráfico 8: ¿Que tan importantes son los Conocimientos Aeronáuticos para el profesional de este rubro?

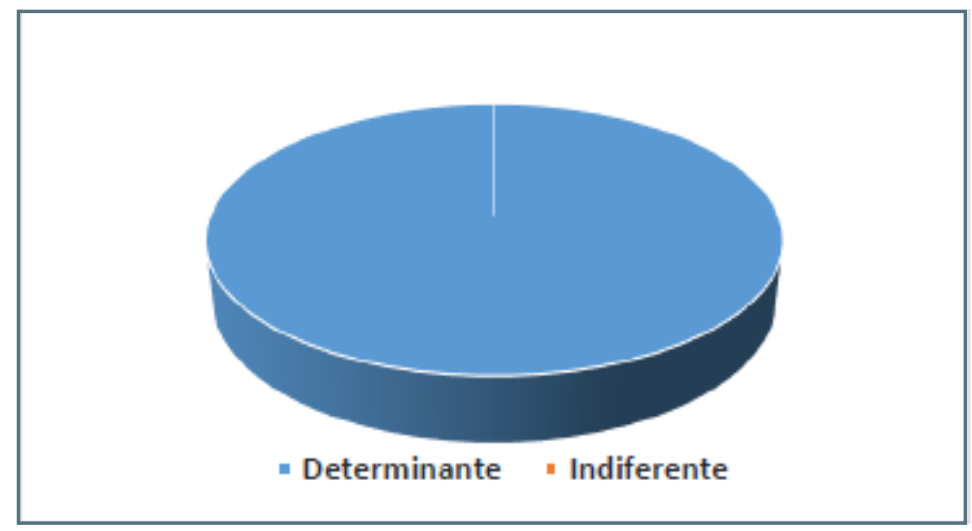

Fuente: Elaboración propia en base al Documento Bases.

En la formulación de esta pregunta se entiende el que el término conocimientos significa el manejo por parte del profesional aeronáutico de las tecnologías aeronáuticas, la infraestructura aeroportuaria y operaciones aéreas. Hay conciencia de que el manejo de estas áreas complementa y refuerza los conocimientos básicos que normalmente se manejan para el desempeño cotidiano de los técnicos y profesionales activos en el rubro.

Gráfico 9: ¿Dónde recibió Usted su entrenamiento Aeronáutico?

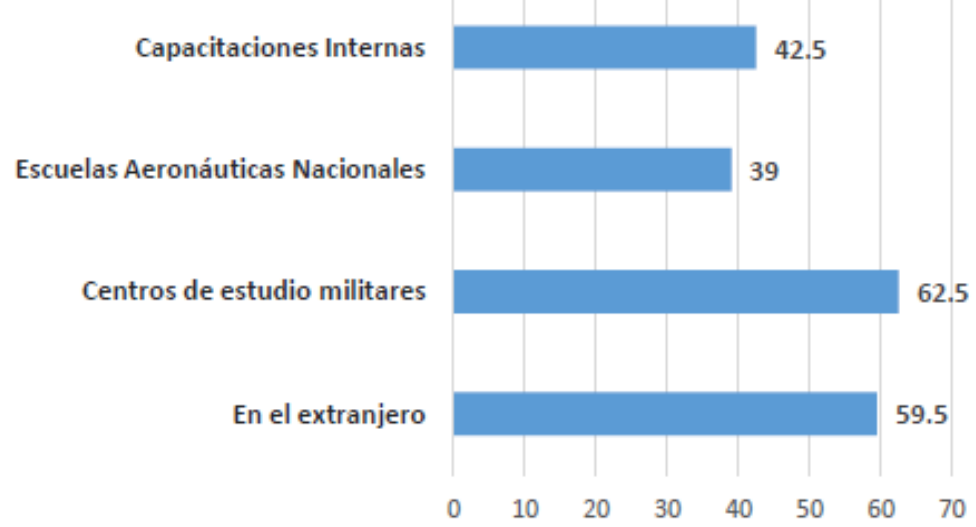

Fuente: Elaboración propia en base al Documento Bases. 
Tiene consecuencias importantes el hecho constatado por la respuesta a esta pregunta, no se puede esperar un gran arraigo de nuestros técnicos y profesionales, si la mayoría de ellos ha sido formado en el extranjero y tampoco se pueden esperar de ellos un conocimiento profundo de la aeronáutica civil cuando la mayoría de ellos ha sido formado en la aviación militar.

Gráfico 10: ¿Le interesaría a su empresa contratar personal Aeronáutico egresado de la Universidad?

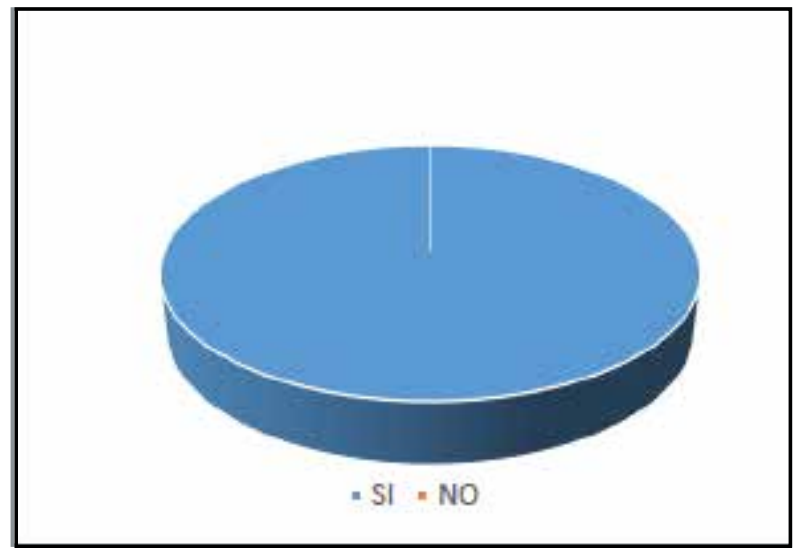

Fuente: Elaboración propia en base al Documento Bases.

En este caso los entrevistados fueron funcionarios del más alto nivel en sus empresas y organizaciones, con lo cual la total apertura para aceptar como parte de su personal egresados universitarios, debe ser tomada en cuenta para fortalecer los lazos entre la Universidad y los sectores públicos y privados del País. Una posibilidad para fortalecer estos vínculos podría ser la suscripción de convenios que fortalecieran los procesos formativos y abrieran campos de trabajo a los egresados de los procesos formativos impulsados desde la UNAH.

\section{Conclusiones y recomendaciones}

\section{Conclusiones}

- Este trabajo de investigación ha resultado ser para quienes colaboramos en él, una la experiencia nueva y útil, puesto que, por una parte, ha representado una primera oportunidad para reflexionar sobre el presente y el futuro de la Aeronáutica Civil hondureña en la perspectiva de la Educación Superior Civil, 
por otra, ha llamado la atención sobre temas de vital importancia que deberán ser retomados en clave de colaboración con los distintos sectores que trabajan en el rubro aeronáutico en Honduras.

- Partiendo de lo que se conoce el campo aeronáutico las propuesta de esta investigación se considera innovadora, puesto que inaugura en el País una perspectiva de análisis eminentemente civil, desde la cual se puede abordar la problemática de la aeronáutica civil en el contexto de un amplio espectro del desarrollo económico y social del País.

- Los datos, información y análisis aportados por esta investigación ofrecen una buena base para tomar decisiones sobre la problemática en el sector aeronáutico nacional el cual para su sobrevivencia tienen que hacer un esfuerzo importante para poder aportar profesionales y técnicos a un mercado global con una demanda creciente, pero también con grandes exigencias.

- Las instituciones y organismos miembros del equipo interinstitucional mantenemos la sensación de haber realizado un trabajo de calidad con aportes relevantes para el desarrollo de la aeronáutica como producto de un involucramiento coordinado de los distintos actores comprometidos con el sector.

- Se han detectado las grandes carencias en el sector aeronáutico; pero al mismo tiempo se han descubierto grandes oportunidades de acción, que solo pueden ser aprovechadas con el aporte necesario y oportuno de la Educación Superior.

- La diferenciación del campo militar con respecto del campo civil, establecido con el Convenio de Chicago de 1944, fortalece a los profesionales de ambos campos, pues ofrece la oportunidad para que cada uno se empodere y desarrolle su propio campo en la perspectiva que le es propia.

- Consideramos lo más relevante de la investigación, el SI del 100 por ciento de la población hondureña consultada a la pregunta sobre la necesidad de contar en la UNAH con un Departamento de Ciencias Aeronáuticas, puesto que es un indicativo de la necesidad existente en el campo de la aeronáutica civil del concurso de la Academia Civil.

- La propuesta que mejor se adapta a las necesidades de formación y de participación de la UNAH en el campo aeronáutico, es la de una Licenciatura en Ciencias Aeronáuticas que dé cuenta de la demanda de profesionales y técnicos existente en el País, en la región y en el mundo. 


\section{Recomendaciones}

- Se debe intensificar el trabajo con la DGAC Y COCESNA, ya que este relacionamiento interinstitucional será la base para abrirse a nuevas alianzas, por ejemplo por medio de la DGAC, se pueden establecer coordinaciones con la $\mathrm{OACl}$ y por esta vía se puede iniciar el proceso de mejora que nos conducirá al tan añorado certificado que nos avale como país de calidad en los requisitos para la aeronavegabilidad mundial.

- Se debe contar con una agenda definida en torno a la cual se establecerían las distintas alianzas con universidades y centros de formación en materia de aeronáutica; es recomendable que estas alianzas sean ratificadas mediante convenios como acostumbra la UNAH.

- Una de las primeras prioridades para la conformación del equipo profesional que continúe con el trabajo de conformación del Departamento de Ciencias Aeronáuticas dentro de la FACES, es la asignación del personal que van a ofrecer la UNAH, la DGAC y ADAEH.

- El montaje y desarrollo de Diplomado, seminarios, conferencias, talleres, foros y otras formas de educación no formal superior, se debe aprovechar como una oportunidad para explorar el campo de la Aeronáutica civil; partiendo de los hallazgos que ya aporta el estudio de la oferta y la demanda de las Bases para el establecimiento de las ciencias aeronáuticas, se puede ahondar sobre las necesidades reales en este campo, los candidatos para la licenciatura en Ciencias Aeronáuticas, los temas de estudio más pertinentes, temas para nuevos para la oferta académica, entre otros.

- Desde las propuestas que se hagan en materia de educación no formal (diplomados, ciclos de conferencias, seminarios, talleres) se deben combinar aspectos técnicos de la ciencias aeronáuticas con aspectos administrativos que le sirven de soporte y le ayuden a conocer el campo aeronáutico en un espectro más amplio.

- Dado lo novedoso del campo de la Aeronáutica, durante la elaboración del Diagnóstico para la Carrera se recomienda se le dé mayor prioridad como instrumento de consulta a las entrevistas estructuradas con informantes claves; esto sin menoscabo del manejo de datos cuantitativos que puedan ser obtenidos en fuentes secundarias y mediante la aplicación de encuestas. 
- La colaboración con entes regionales e internacionales vinculados a los temas de las ciencias aeronáuticas, será vital durante el proceso de afianzamiento de las ciencias aeronáuticas en un país como Honduras, en donde el tema tiene fuerte raigambre militar, en tal sentido se debe involucrar esta colaboración para el desarrollo de las clases, talleres y conferencias de las primeras promociones del Diplomado y la Licenciatura en Ciencias Aeronáuticas.

\section{Bibliografía}

- Haslett, C. (2008). Essentials of Radio Wave Propagation. Ofcom, UK: Cambridge University Press.

- Richards, J. A. (2008). Radio Wave Propagation An Introduction for the Non-Specialist. Canberra, Australia: Springer.

- Aeropuertos de Honduras. (2013). Reporte estadístico mensual. Tegucigalpa: No publicado.

- Aeropuertos de Honduras SA. (2012). Manual de procedimiento de cobro por servicios aeroportuarios. Tegucigalpa, Francisco Morazán , Honduras: No publicado.

- Alberto Colindres, A. M. (2014). Cuerpo de Contenido para la Formación de Controlador de Tránsito Aéreo en el ámbito de las ciencias aeronáuticas en Honduras. Tegucigalpa: No publicado.

- American Association of Variable Star Observers (AAVSO). (14 de Junio de 2012). Sudden lonispheric Disturbances (SIDs). Recuperado el 08 de 11 de 2012, de American Association of Variable Star Observers (AAVSO): http://www.aavso.org/ solar-sids

- American Meteorological Society. (2012). Glossary of meteorology. Allen Press.

- Castillo, Y. (2012). Diseño de un radiotelescopio para actividades de investigación . Revista de Ciencias espaciales, --.

- Chernan, C. (1978). The handbook of solar flare monitoring \& propagation forecasting. Blue rdge, USA: Tab Books.

- Committee on the Societal and Economic Impacts of Severe Space Weather Events. (2008). Severe Space Weather Events - Understanding Societal and 
Events: A Workshop. Washington, DC, United States of America: The National academy Press.

- Cummer, S., Inan, U., \& Bell, T. (Noviembre-Diciembre de 1998). lonospheric D region remote sensing using VLF radio atmpspherics. Radio Science, 33(6), 17811792.

- Department of the Navy Naval Electronic Systems Command. (1972). Naval Shore Electronic Criteria: VLF, LF, and MF Comunication Systems. Washington: U.S. Printing Office.

- DGAC. (2004). Ley de Aeronáutica Civil. Tegucigalpa: DGAC.

- DGAC Chile. (22 de Noviembre de 2012). DGAC. Obtenido de www.dgac.gob.cl/ portal

- Dixon, B. (1991). " La Arquitectura del periodo Formativo y la competencia del estado social en Yarumela, Honduras. En YAXKIN. Honduras .

- Dixon, B. (2008). Yarumela: una historia de investigacion arqueologica en el sitio y su lugar en la antigua historia hondureña. En R. YAXKIN, Revista del instituto hondureño de antropologia e historia (Vol. XXIV, pág. 199). Tegucigalpa, Honduras: ENAG.

- Embry Riddle. (2012). Undergraduate/Graduate Catalog. Daytona: Embry Riddle.

- FUMEC. (27 de Noviembre de 2012). Universidade FUMEC. Obtenido de www. fumec.br/cursos

- Grubor, D., Suli, D., \& Zigman, V. (2005). Influence Of Solar X-Ray Flares On The Earth-lonosphere Waveguide. Serbian Astronomical Jounal, 29-35.

- Haridas, D., Soman, K. P., \& Sundaram, S. (2013). lonospheric Disturbances due to Solar Ionospheric Disturbances due to Solar. International Journal of Advanced Electrical and Electronics Engineering, 69-74.

- Hunsucker, R. D., \& Hargreaves, J. K. (2003). The high-latitude ionosphere and its effects on radio propagation. Cambridge, UK: Cambridge University Press. 
- Interairport, Estado de Honduras. (23 de septiembre de 2000). Contrato de concesiones. Tegucigalpa, Honduras: La Gaceta.

- J.S. Perez, E. M. (1999). Manual Sanitario para Tripulantes de Cabina de Pasajeros. Madrid: Arán Editores.

- Kolarski, A., Grubor, D., \& Suli, D. (8 de Agosto de 2011). Diagnostics Of The Solar X-Flare Impact On Lower lonosphere Through The VIf-Naa Signal Recordings. Baltic Astronomy, 20, 591-595.

- Lang, J.-P. (10 de 05 de 2013). GNU Radio. Obtenido de Welcome to GNU Radio!: http://gnuradio.org/redmine/projects/gnuradio/wiki

- Lashley, J. (2010). The Radio Sky and How to Observe It. (M. Inglis, Ed.) New York, USA: Springer.

- Leech, M. (28 de 03 de 2014). Proyecto SIDSuite. Obtenido de The Comprehensive GNU Radio Archive Network: https://www.cgran.org/browser/projects/SIDSuite

- Mandeville, J. (1986). "Proyecto Arqueologico Valle de Comayagua: Investigaciones en Yarumela-Chical. IX N²,1986, 17-41.

- Mandeville, J. (1997). "Programa Arqueologico de Yarumela, temporada 1983: descubrimientos y analisis". XV, edicion extraordinaria 1990-1994, 5-18.

- More, C., Sharma, A., Bhonsle, R., \& Lynn, K. (2010). Fiel Strength Measurement of VLF Waveguide of VLF RadioWave Propagation at $19.8 \mathrm{KHz}$ betwen Australia and India. Australian Space Science Conferene Series (págs. 249-262). Sydney: Wain.

- Nakamura, S. (1991). Desarrrollo y decaimiento en la periferia de Copán". annals of Latin American studies, 39-95.

- National Bureau of Standards. (1937). Radio Instruments and Measurements. Washington: GOVERNMENT PRINTING OFFICE.

- NOAA/Space Weather Prediction Center. (8 de Octubre de 2012). Space Weather Prediction Center. Recuperado el 27 de Octubre de 2012, de http://www.swpc. noaa.gov/SolarCycle/index.html 
- OACI. (2006). Doc 9562 Manual sobre los aspectos económicos de los aeropuertos, Segunda Edición. Montreal: OACl.

- OACl. (2011). Estudio de la OACI revela gran demanda de personal aeronáutico cualificado de aquí al Año 2030. OACl.

- OACl. (20 de Noviembre de 2012). ICAO. Obtenido de icai.int/safety/dangerous good

- radiosky.com. (30 de 10 de 2013). Radio-SkyPipe II - An Internet Enabled Strip Chart Recorder. Obtenido de Radio-SkyPipe II - An Internet Enabled Strip Chart Recorder: http://www.radiosky.com/skypipeishere.html

- Raulin, J., Berton, F., Gavilán, H., Guevara, W., Rodriguez, R., Fernandez, G., . . . Hadano, R. (2010). Solar flare detection sensitivity using the South America VLF(SAVNET). Journal Of Geophysical Research, 115, A07301.

- Raulin, J., Correia de Matos, P., Hadano, R., Saraiva, A., Correia, E., \& Kaufmann, P. (2009). The Souh America VLF Network (SAVNET):Development installation status, first results. Geofísca Internacional, 48(3), 253-261.

- Sampieri, H. (2010). Metodología de la investigación. México: McGraw Hill.

- spaceweather.com. (s.f.). The Classification of X-ray Solar Flares. Recuperado el Octubre de 2010 de 28, de http://spaceweather.com: http://spaceweather.com/ glossary/flareclasses.html

- U S P M. (27 de Noviembre de 2012). Universidad San Martín de Porres. Obtenido de www.usmp.edu.pe/ffia/aeronauticas

- UKRAA. (30 de 10 de 2013). UK Radio Astronomy Association. Obtenido de UK Radio Astronomy Association: http://www.ukraa.com/www/

- Universidad Técnica Federico de Santa María. (27 de Noviembre de 2012). Ingeniería en aviación comercial. Obtenido de www.usm.edu.cl/admision

- USAC. (s.f.). Atlas Arqueologico de Guatemala. (D. G. Natural, Productor) Obtenido de http://www.atlasarqueologico.com/. 
- Valladarez, O. A. (Septiembre de 2008). Yarumela: Revalorizacion un sitio aqueológico en el valle de Comayagua. (ENAG, Ed.) YAXKIN, XX1V, No1, 211-2226.

- Vivanco, M. (2005). Muestreo estadístico, diseño y aplicaciones. Santiago.

- Wolf, D. (30 de 10 de 2013). Audio Spectrum Analyzer . Obtenido de Audio Spectrum Analyzer : http://www.qsl.net/dl4yhf/spectra1.html 